\title{
Wirtschaftssoziologie und Gesellschaftsanalyse
}

\section{Gertraude Mikl-Horke}

Zusammenfassung: Die Wirtschaftssoziologie der Gegenwart, die sich seit den 1980er Jahren von den USA aus ausgebreitet hat, ist lange Zeit vornehmlich auf die empirische Erforschung des Unternehmenshandelns in Märkten und die Einbettung desselben in die sozialen Beziehungen zwischen den Firmen fokussiert gewesen. Im europäischen sozialwissenschaftlichen Kontext wurde zwar den Institutionen auch Beachtung geschenkt, aber doch aus der Sicht der Wirkungen derselben auf das Markthandeln. Die Wirtschaftssoziologie schien den methodologischen Individualismus der Mikroökonomie übernommen zu haben und wurde von manchen eher als eine Abart der neoliberalen Ökonomie betrachtet. Der Beitrag argumentiert, dass das Verständnis von Wirtschaft über den Markt hinaus erweitert und wieder stärker auf die Gesellschaft und die Folgen der wirtschaftlichen Handlungen für diese bezogen werden muss. Dabei ist in der Gegenwart das Problem der Verteilung von Einkommen und Vermögen besonders wichtig, das in der Soziologie durch die Überbetonung der sozialen Wertschätzung, von Status und Prestige, ,entmaterialisiert" wurde. Die ökonomische Ungleichheit als solche ist ein Grundproblem für die Gesellschaft und für die Soziologie. Letztere muss über die empirische Feststellung von Strukturunterschieden hinaus den Markt als einen politisch-kulturellen Tatort umdeuten und ihr eigenes Verständnis von Wirtschaft als soziologisches Erkenntnisobjekt finden.

Schlüsselwörter: Neue Wirtschaftssoziologie, Marktsoziologie, soziale und ökonomische Ungleichheit, Gesellschaftsanalyse

G. Mikl-Horke $\square$

WU - Wirtschaftsuniversität Wien, Institut für Soziologie und Empirische Sozialforschung Welthandelsplatz 1, Gebäude D4, 1020 Wien, Österreich

E-Mail: Gertraude.Mikl-Horke@wu.ac.at 


\title{
Economic sociology and societal analysis
}

\begin{abstract}
The economic sociology that developed from the 1980s onwards starting in the US, focussed on the empirical research on actions of firms in markets and their embeddedness in interfirm social relations. In the social science context in Europe institutions had been paid attention as well, but mostly in view of their effects on market behaviour. Economic sociology seemed to have embraced the methodological individualism of microeconomics and was seen by some even as a derivative of neoliberal economics. The article argues that the concept of the economy must be enlarged beyond the market and oriented more strongly at society and the social consequences of economic actions. In this connection the problem of economic distribution is especially important at present, but had been ,immaterialised“ in sociology by an overemphasis on differences of social status and prestige. The inequality of income and wealth must itself be understood as a fundamental problem for society and for sociology, and the latter must go beyond studying structural differences by empirical research and reformulate the market as a political-cultural arena of action and find her own conception of the economy as sociological object of cognition.
\end{abstract}

Keywords: New economic sociology, market sociology, social and economic inequality, societal analysis

\section{Einleitung}

Dass Wirtschaft ohne Gesellschaft, aber auch Gesellschaft ohne Wirtschaft nicht denkbar sind, davon kann man sich durch einen Blick auf die Entwicklung der menschlichen Kultur und Geschichte leicht überzeugen. In den Denkweisen und Konzepten der modernen Sozialwissenschaft erscheint jedoch gerade diese Verbindung von Wirtschaft und Gesellschaft problematisch, sind beide doch Erkenntnisobjekte voneinander getrennter Einzelwissenschaften. Zwar gab es immer wieder Versuche, die Trennung von Ökonomie und Soziologie zu überwinden, und in der Gegenwart stellt die Wirtschaftssoziologie, die sich seit den 1980er Jahren von den USA aus entwickelt und verbreitet hat, einen derartigen Versuch dar wie auch die Diskussion um die Sozio- bzw. Sozialökonomie (vgl. Mikl-Horke 2011).

Die Wirtschaftssoziologie der Gegenwart ist weitgehend auf die empirische Untersuchung des Handelns von Unternehmen und der sozialen Beziehungen auf Märkten fokussiert und nimmt die weiteren gesellschaftlichen Bezüge im Wesentlichen aus der Perspektive der Wirkungen von Institutionen auf das wirtschaftliche Handeln wahr. Hingegen setzt sie sich wenig mit gesellschaftstheoretischen Fragen auseinander, noch hinterfragt sie systematisch das auf „den Markt“" reduzierte Verständnis von Wirtschaft. Dieser Beitrag soll darauf hinweisen, dass eine gesellschaftstheoretische und -analytische Perspektive für eine Wirtschaftssoziologie, die Soziologie sein will, wichtig ist. Denn Annäherung oder Integration der Sozialwissenschaften 
bedeuten nicht einseitige Assimilierung, sondern den Diskurs auf der Basis der jeweils eigenen Objektdefinitionen und Erkenntnisziele.

Zunächst wird in aller Kürze auf die Entwicklung der Wirtschaftssoziologie und ihr Verhältnis zur Ökonomie eingegangen. Im Anschluss daran wird argumentiert, dass Wirtschaftssoziologie den Zusammenhang zwischen Gesellschaftsstruktur und Wirtschaft berücksichtigen muss, und dabei in der Gegenwart dem Problem der ökonomischen Ungleichheit im Gefolge der Dominanz der globalen Finanzmärkte besondere Bedeutung zukommt.

\section{Wirtschaftssoziologie - Soziologie oder Ökonomie?}

Das auf Marktgesetz und rationalem Nutzenkalkül beruhende Konzept der Wirtschaftstheorie übte auf das allgemeine Verständnis von Wirtschaft - und auch jenes der Soziologen - eine beträchtliche Faszination aus, die auch dazu führt, dass dieses oft mit der Wirklichkeit identifiziert wird. Sogar Weber meinte in Bezug auf die abstrakten Annahmen der Wirtschaftstheorie, dass sich die realen Verhältnisse dem Modell asymptotisch annähern würden (vgl. Maurer und MiklHorke 2015, S. 57 ff.). Weber bezog sich dabei vor allem auf die österreichische Version der Grenznutzentheorie, in Parsons“ und Smelsers „Economy and Society“ (1956), einem weiteren Markstein in der Entwicklung der Wirtschaftssoziologie, war es dann die neoklassisch-keynesianischen Synthese, die die Grundlage für das Wirtschaftsverständnis darstellte.

Auch die empirischen Studien der zweiten Hälfte des 20. Jahrhunderts, die sich vornehmlich mit Konsum, Arbeit und Organisation beschäftigten, hinterfragten die von der Ökonomie und ihrer Popularisierung ins Leben gesetzte Vorstellung von Wirtschaft als Markt nicht. Soziologen kritisierten nur die fehlende - von der Wirtschaftstheorie ohnehin nicht beabsichtigte - Realitätsnähe des neoklassischen Verhaltensmodells, meinten damit aber die Nicht-Berücksichtigung der als vorwiegend nicht-rational verstandenen sozialen Motive und Verhaltensweisen der Konsumenten und der Arbeitenden. Sie lieferten ihrerseits empirische Untersuchungen über Verhaltens- und Einstellungsmuster, was Anerkennung fand in einer Zeit, die durch wohlfahrtsstaatliche Institutionen und eine auf die Förderung der Massennachfrage abzielende Wirtschaftspolitik charakterisiert war. Unter diesen Voraussetzungen waren Politik und Unternehmen an empirischen Untersuchungen als Informationsquellen über Einstellungen und Verhaltensweisen von Arbeitnehmern, Konsumenten und Wählern sehr interessiert, was die Stellung der Soziologie in der akademischen Welt und ihren Einfluss als Beraterin der Politik förderte.

Wirtschaftssoziologie bedeutete in dieser Epoche bis zu den 1980er Jahren vor allem eine Bezeichnung für einen Sammelbereich der empirischen Forschungsbereiche, die sich mit den „sozialen“ Dimensionen in der Wirtschaft beschäftigen, d.h. mit den Verhaltensweisen und Einstellungen von Arbeitenden und Konsumenten; gelegentlich war das auch mit dem Versuch, den Begriffskanon der modernen Soziologie auf die Wirtschaft zu übertragen, verbunden. Seit etwa 
1980 entstand dann die noch heute oft als „neue“ bezeichnete Wirtschaftssoziologie (Maurer und Mikl-Horke 2015, S. 141 ff.). Dies koinzidierte zeitlich mit dem politischen Umschwenken zu marktliberalen Auffassungen und „,angebotsorientierten“ Wachstumsstrategien in USA und UK. Diese politischen Strategien veränderten die wirtschaftliche Realität nachhaltig und führten u.a. dazu, dass in der Gegenwart wirtschaftliche Themen und Probleme im Vordergrund des öffentlichen Interesses stehen, was diese auch für die sozialwissenschaftliche Forschung interessant machte.

In der Ökonomie ihrerseits war das Bewusstsein gewachsen, dass institutionell-politische Bedingungen Folgen für die Wirtschaft haben und dass Markthandeln Kosten verursacht. Es kam daher zu einer stärkeren Berücksichtigung der Normen, der Institutionen und der Kultur, was dem Neo-Institutionalismus, der allerdings keine Abkehr von der Grenznutzen- und Markttheorie darstellt, Auftrieb gab (vgl. Schmid und Maurer 2003). Auch beanspruchten neoklassische Theoretiker, dass das Rational-Choice-Modell über die Wirtschaft und den Markt hinaus auch für die Erklärung des sozialen und des politischen Handelns Geltung habe. Das wurde innerhalb der Soziologie von jenen aufgegriffen, die auch soziales Handeln als rational erkannten, und führte zur Konstitution einer ökonomischen Soziologie im Sinne einer „,rational reconstruction of society“ (Coleman 1993).

Im Gegenzug gaben einige Soziologen ihre Zurückhaltung in Bezug auf den zentralen Gegenstandsbereich der Ökonomie auf und begannen sich gezielt mit Markt und Preisbildung zu beschäftigen. Schon Hans Albert hatte in den 50er Jahren eine Marktsoziologie vorgeschlagen; diese sollte eine empirisch-analytische Wissenschaft sein und die Modelltheorie der Ökonomie ersetzen (Albert 2014). Die neue Wirtschaftssoziologie setzt auch am Markt an und beansprucht diesen als legitimen Gegenstand der Soziologie, wobei dieser aber weitgehend so aufgefasst wird, wie er von der Ökonomie bestimmt wird, und überdies mit Wirtschaft weitgehend gleichgesetzt wird.

Die Wirtschaftssoziologie beruft sich auf einen Aufsatz von Mark Granovetter (1985), worin dieser feststellte, dass soziale Beziehungen für die Erklärung wirtschaftlicher Sachverhalte und Prozesse von zentraler Bedeutung sind. Er kritisierte sowohl über- als auch untersozialisierte Sichtweisen, wie sie die strukturfunktionalistische Soziologie Talcott Parsons einerseits, die ökonomischen Ansätze, auch jene der neo-institutionalistischen Ökonomie, andererseits repräsentieren. Er plädierte für eine Analyse, die sowohl von den individuellen Motiven und Handlungen als auch von deren Einbettung in soziale Beziehungen und Institutionen ausgehen sollte. Die angebotsorientierte mikroökonomische Perspektive wird jedoch insofern übernommen, als in vielen Studien die Unternehmen bzw. ihre Vertreter die zentralen Akteure des Marktes darstellen, d.h. ihr Handeln bestimmt Entstehung und Wandel der Märkte. In einem ebenfalls grundlegenden Beitrag hatte Harrison White (1981) argumentiert, dass Firmen sich nicht primär an ihren Schätzungen in Bezug auf eine amorphe Nachfrage orientieren, sondern an den 
Strategien anderer Firmen, teils um erfolgreiche Firmen nachzuahmen, teils um für sich eine Marktnische zu finden.

Das Handeln der Akteure auf Märkten wird als sowohl sozial als auch rational motiviert verstanden, wobei besonders betont wird, dass auch das Markthandeln der Unternehmen sich an sozialen Beziehungsaspekten orientiert. Die Wirtschaftssubjekte, die Firmen bzw. ihre Vertreter, handeln rational, aber auch im Rahmen und mit bewusstem Bezug auf die sozialen Beziehungen, die zwischen ihnen bestehen. Ronald Burt (1992) untersuchte die Formen, die Netzwerkbeziehungen annehmen können. Er wies darauf hin, dass sie als Sozialkapital eingesetzt werden, um positive wirtschaftliche Ergebnisse zu erzielen. Vielfach dient der Hinweis auf die ökonomische Rationalität des Sozialen zur Begründung der Relevanz soziologischer Forschung für Wirtschaft und Politik. Granovetter seinerseits betonte allerdings, dass die Menschen den sozialen Aspekten über die instrumentellen Effekte hinaus auch in Wirtschaftsbeziehungen eine eigene Wertigkeit beimessen (Granovetter 2005).

Der Aufstieg der Wirtschaftssoziologie schlug sich in zahlreichen empirischen Studien über spezielle Märkte und ihre Einbettung in die Netzwerke sozialer Beziehungen nieder. Der Fokus verschob sich, nachdem dieser Ansatz auch in Europa Fuß gefasst hatte, in Richtung auf die stärkere Betonung der institutionell-kulturellen Aspekte. Dennoch spielen Märkte und das Handeln von Unternehmen auch hier eine zentrale Rolle in der Wirtschaftssoziologie, und Normen, Institutionen und soziale Aspekte werden vielfach in Bezug auf ihre Wirkungen auf das Markthandeln der Firmen untersucht. Als Ansatzpunkt einer soziologischen Analyse der Wirtschaft bietet sich auch insbesondere das Problem der Ungewissheit in Märkten an (vgl. Beckert 1996). Das Wissen über Normen, Regeln, Institutionen, Konventionen und soziale Beziehungen kann die Ungewissheit reduzieren; damit tritt ihre Funktion für die Effizienz des Marktes und den Wirtschaftserfolg der Unternehmen zutage, welchen die Beseitigung von Ungewissheit jedoch auch hindern kann, wenn sie dadurch die unternehmerische Initiative und Risikobereitschaft mindert.

Aus dieser kurzen Skizze wird deutlich, dass die Wirtschaftssoziologie in dieser Form weitgehend auf die Erforschung von Märkten und aus der Perspektive der privatwirtschaftlichen Aktivitäten betrieben wird. Die Berücksichtigung sozialer Elemente aber findet sich auch bei vielen Ökonomen, insbesondere in empirischen, transdisziplinären und heterodoxen Ansätzen (vgl. Bögenhold 2011). Der soziologische Charakter der Marktsoziologie erscheint problematisch durch die teilweise Übernahme der mikroökonomischen Perspektive bzw. des methodologischen Individualismus und die doch starke Betonung der wirtschaftlichen Funktionalität sozialer Aspekte, von Institutionen, sozialen Beziehungen und Netzwerken. Manche sehen in dieser neuen Wirtschaftssoziologie daher nur eine Ökonomie mit anderen Mitteln. Insofern fügt sich die neue Wirtschaftssoziologie allerdings auch in die seit Weber, Pareto und Parsons bestehende Tradition ein, die sich mit ihrer Soziologie eigentlich an die Ökonomen gewandt hatten. 


\section{Wirtschaft und Gesellschaft: Für eine gesellschaftsanalytische Perspektive der Wirtschaftssoziologie}

Eine soziologische Forschung, die vornehmlich am Markt ansetzt, kann nicht als erschöpfend für eine Wirtschaftssoziologie betrachtet werden, ist in gewisser Weise auch ein seltsames Unterfangen für eine Soziologie. Zwar verweisen die neuen Wirtschaftssoziologen darauf, dass sie eben nicht den Markt, sondern die Märkte in ihrer Vielfalt untersuchen (vgl. Aspers 2011; Beckert, Diaz-Bone und Ganßmann 2007). Doch das tun Ökonomen auch, wenn sie sich mit den verschiedenen Sparten der Wirtschaftstätigkeit beschäftigen. Ökonomen sind sich auch durchaus bewusst, dass zwischen Markt und Wirtschaft unterschieden werden muss, denn zum einen wirkt der Markt auf der Grundlage der politischen, institutionellen und kulturellen Bedingungen. Der Markt bzw. die Märkte umfassen auch nicht die Gesamtheit der Wirtschaft, aber obwohl neben der Staatswirtschaft auch der Dritte Sektor und die Sozialwirtschaft gegenwärtig wieder mehr Beachtung finden, werden sie meist als Randerscheinungen bzw. als Supplemente der kapitalistischen Marktwirtschaft gesehen.

Wenn man Befunde von Wirtschaftsanthropologen und Wirtschaftshistorikern berücksichtigt, erkennt man, dass Wirtschaften zu allen Zeiten und in allen Kulturen nicht durch ein einheitliches Prinzip geleitet war, sondern sich als vielschichtig, vielfältig und widersprüchlich darstellte. Das wussten etwa die Philosophen der griechischen Antike wie Aristoteles, der zwischen „chrematistike“ (Bereicherungskunst) und „oikonomia“ (Haushaltungskunst) unterschied, sehr wohl (vgl. Mikl-Horke 2008, S. 7 ff.). Erst die klassische Nationalökonomie mit ihrer Idee der Harmonie von individuellen Interessen, Moralordnung und Staatsfinanzierung schuf die Vorstellung einer Einheit, versprach Kontrolle ohne Zwang und die Legitimierung des Gewinnstrebens durch seine Funktion für die Bedarfsdeckung der Gesellschaft und den „common wealth“ der Nation. Damit wurden der Tausch und seine Voraussetzung, die Arbeitsteilung, zum zentralen Prinzip des Wirtschaftens, und dies führte schließlich zur Deutung moderner Wirtschaftsweise als Markt kulminierend in dem fiktiven Modell der vollkommenen Konkurrenz. Das moderne Verständnis der Wirtschaft wurde durch dieses epochale Programm, das gleichzeitig eine ideale Gesellschaftsvorstellung enthielt, die sich politisch mit dem Konzept der Demokratie verband, geprägt und mit Hilfe der Verwissenschaftlichung wirtschaftlicher Berufe und der Verbreitung der Marktrhetorik in Praxis umgesetzt (vgl. Ötsch 2009). Damit trat das Wissen, dass Wirtschaften immer durch die Spannung zwischen Bedürfnisbefriedigung und Vorteilsstreben, zwischen Hunger und Profit bestimmt ist, in den Hintergrund. Wirtschaft war niemals ein System, das durch ein einziges Prinzip oder einen Mechanismus bestimmt war, sondern vielmehr durch unterschiedliche Ziele der Bedarfsdeckung und der Bereicherung repräsentiert; sie war auch niemals nur friedlich, vielmehr über den Großteil der Geschichte eng mit Krieg, Eroberung und Beute verbunden. 
Die Soziologie kann nicht einfach das Verständnis von Markt und Wirtschaft von der Ökonomie übernehmen, da sie andere Erkenntnisziele verfolgt als diese. Nicht nur muss sie es hinterfragen und erweitern, sondern sich auf ihre eigenen Problemstellungen und Erkenntnisziele besinnen. Diese liegen in dem Zusammenwirken von Wirtschaft und Gesellschaft begründet, was auch impliziert, dass sie die makrosoziale Dimension wieder stärker berücksichtigen muss. Sehr richtig fordert daher Beckert, „Wirtschaftssoziologie als Gesellschaftstheorie“ zu begreifen (Beckert 2009a).

Die Perspektive auf den Zusammenhang von Wirtschaft und Gesellschaft rückt auch den Begriff des Kapitalismus wieder ins Blickfeld der Soziologie. Karl Marx hatte die kapitalistischen Produktionsverhältnisse als eine Gesellschaftsformation verstanden, in der Akkumulation und Dynamik durch die Umwandlung von G-W-G` bestimmt sind. Damit hatte er dem Geld und dem Profitstreben besondere Bedeutung zuerkannt. Georg Simmel, Max Weber und Werner Sombart haben den Kapitalismus dann primär als Ausprägung der Individualisierung und Rationalisierung der Kultur und des Aufstiegs des Wirtschaftsbürgertums verstanden. Insbesondere Weber hatte aber das Charakteristikum des modernen industriellen Kapitalismus auch in der Kapitalrechnung gesehen, und die Börse als die rationalste und damit idealtypisch repräsentativste Institution des modernen Kapitalismus charakterisiert (vgl. Weber 2000). Diese Betonung des Geldes und seiner Funktion als Kapital trat jedoch in der Soziologie dann gegenüber den technisch-industriellen Veränderungen der Organisation der Produktion und den Formen der Arbeit in den Hintergrund.

Die Soziologie orientierte sich an der Struktur und Ordnung der Industriegesellschaft, während das Geld als eines der symbolisch generalisierten Kommunikationsmedien definiert verallgemeinert und „entökonomisiert“ wurde (Deutschmann 2008, S. 42; vgl. Mikl-Horke 2014). In der zweiten Hälfte des 20. Jahrhunderts beschäftigten sich selbst kritische Theoretiker daher mehr mit Problemen der Technokratie, Bürokratie und Entfremdung als mit dem Kapitalismus als Gesellschaftsform und mit der Rolle von Geldbesitz, -verteilung und -verwendung. Erst spät nach dem Einsetzen der Wirkungen der neoliberalen Wende kam es zu einer Renaissance des Kapitalismusbegriffs und zur Erkenntnis der Bedeutung von Geld und Kapital als „,[...] zentrale Elemente des auf individuelle Autonomie orientierten Wertesystems moderner Gesellschaften [...]“ (Deutschmann 2008, S. 173).

Arbeit einerseits, Geld andererseits stellen die Perspektiven für wirtschaftssoziologische Reflexion dar (vgl. Ganßmann 1996), was sich mit der grundsätzlichen Widersprüchlichkeit der Wirtschaft zwischen Bedarfsdeckung und Bereicherung, wie sie Aristoteles erkannte, verbinden lässt. Wie Arrighi (2002) aufzeigt, weist diese auch eine historische Zyklizität auf, indem sich Phasen der Dominanz materieller Produktion mit jenen monetär-finanzieller Dominanz abwechseln; er konstatiert eine ,[...] alternation of epochs of material expansion with phases of financial rebirth and expansion...In phases of material expansion money capital ,sets in motion ${ }^{\circ}$ 
an increasing mass of commodities [...]; and in phases of financial expansion an increasing mass of money capital ,sets itself free' from its commodity form, and accumulation proceeds through financial deals [...]" (Arrighi 2002, S. 6).

Der neuen „financial revolution“ des „langen 20. Jahrhunderts“ - und den Nachwirkungen in den ersten Jahrzehnten des 21. Jahrhunderts - kommt jedoch eine besondere Bedeutung angesichts des Volumens und der Geschwindigkeit der Finanzströme zu, was sich auch in dem oft zitierten Begriff des „Finanzmarktkapitalismus“ (Windolf 2005) als Kennzeichnung einer neuen gesellschaftlich-wirtschaftlichen Formation niederschlug. Wie in allen Phasen der finanziellen Dominanz verstärkte sich auch in der Gegenwart die ökonomische Ungleichheit mit all ihren sozialen und kulturellen Folgen, verschärft durch die globale Dimension, die der gegenwärtige Kapitalismus besitzt.

\section{Soziale und ökonomische Ungleichheit}

Wenn man an die Entstehung der Soziologie im Kontext der Transformation der vertikal stratifizierten Gesellschaft denkt, wird deutlich, dass der gesellschaftlichen Ungleichheit eine besondere Bedeutung als eine grundlegende Problemstellung zukommt. Wirtschaftliche Veränderungen im Sinne der Kapitalbildung und der Einkommens- und Vermögensverteilung spielten im Zuge dieser Transformation eine wichtige Rolle. Dieser Aspekt trat jedoch in der sich als Einzelwissenschaft um Anerkennung bemühenden Soziologie im Laufe des 20. Jahrhunderts mehr und mehr in den Hintergrund zugunsten der Erforschung und Erklärung der sozialen Ordnung der modernen Industriegesellschaft als einer positionalen Ordnung auf Grund von erworbenen Statuskriterien wie Beruf und Bildungsniveau. Dabei kam dem Ansehen, der sozialen Wertschätzung eine große Bedeutung zu und der Begriff der Sozialstruktur wurde dadurch weitgehend ,immaterialisiert“, auf Einstellungen und Verhaltensmuster bezogen (vgl. Fürstenberg 1966). Empirische Untersuchungen ließen das Bild einer relativ homogenen Schichtung entstehen und resultierten in den 60er Jahren des vergangenen Jahrhunderts in Befunden einer Mittelschichtgesellschaft. Diese Vorstellung fand eine gewisse reale Grundlage in dieser Epoche, die von den Erwartungen dauerhaften Wachstums und des wohlmeinenden Wirkens des Wohlfahrtsstaates geprägt war. Die Klassenkonzeption im Sinne objektiver Unterschiede ökonomischer Verfügungsmacht schien ihre Erklärungskraft in einer „konsensualen Wohlstandsgesellschaft“ verloren zu haben bzw. in einer „Klassengesellschaft ohne Klassen“ zu resultieren (vgl. Kreckel 1998). Studien über den Wandel des Freizeit- und Konsumverhaltens führten zu Befunden einer Individualisierung der Lebensstile. Ästhetische, kognitive, kulturelle Aspekte wurden zur Beschreibung der modernen Gesellschaft herangezogen; selbst der technische Wandel und das Geld wurden in Begriffen von Kommunikationsflüssen und -medien ausgedrückt. Auch jene Theoretiker, die klassenbedingte Ungleichheit thematisierten, verwiesen weniger auf 
die ökonomischen Unterschiede als auf die Instrumentalisierung von kulturellem, sozialem oder symbolischem Kapital für den Erwerb ökonomischen Kapitals (vgl. Bourdieu 1992).

Die Folge war, dass die ökonomische Ungleichheit in der Soziologie lange Zeit wenig, und wenn überhaupt, dann nur indirekte Beachtung fand (Myles 2003). Oder anders gesagt: Soziologische Gesellschaftsanalyse wurde nicht auf die Verhältnisse und Entwicklungen in Politik und Wirtschaft bezogen (Preglau 2007; Nollmann 2007). Erst allmählich kam es seit den 1990er Jahren wieder zur Erkenntnis der Bedeutung ökonomisch-materieller Unterschiede zwischen Gruppen, Staaten und Gesellschaften, als durch Neoliberalismus, die Expansion und Geschwindigkeit der deregulierten globalen Finanzmärkte und durch die Globalisierungsstrategien der Konzerne eine tiefgreifende Transformation vor sich ging. In der Ökonomie wurde dem Verteilungsproblem, das bisher theoretisch in ein Distributionsproblem des Marktes umformuliert worden war, wieder größere Aufmerksamkeit zuteil.

In empirischen Untersuchungen zum Wandel ökonomischer Ungleichheit zeigte sich, dass sich in längerfristiger Sicht markante Veränderungen in der Verteilung von Einkommen und Vermögen erkennen lassen. Während die Ungleichheit in den letzten Dekaden des 19. Jahrhunderts sehr hoch war, nahm sie im Laufe des 20. Jahrhunderts und mit erheblichen Schwankungen auf Grund von Depression und Kriegen bis in die 70er Jahre ab, um seit den 1980er Jahren wieder anzusteigen (vgl. Goesling 2001). Dieser Anstieg begann besonders stark in den USA, verbreitete sich dann aber auch in Europa. Er war zunächst durch den Anstieg der höchsten Einkommen verursacht, wodurch sich die Differenz zwischen oben und unten in der Einkommenspyramide stark vergrößerte. Es kam zu einer Polarisierung zwischen den Spitzeneinkommen und den niedrigen Einkommen, deren Anteil sich vergrößerte; die mittleren Schichten dazwischen hingegen schrumpften immer mehr (Atkinson 2007; Atkinson, Piketty und Saez 2011). Auf Grund der Veränderung der Beschäftigungsformen zeigte sich zudem eine Verstärkung der Einkommensunsicherheit durch die Zunahme existentiell prekärer Solo-Selbständiger, was Bologna als „Zerstörung der Mittelschichten“ kommentierte (Bologna 2006).

Auch die Europäische Kommission hat ein Forschungsprojekt beauftragt, das die „Growing Inequalities“ Impacts“ (GINI) untersucht. Dabei zeigte sich beim Vergleich der Einkommenunterschiede in den reichen Ländern ein genereller Anstieg der ökonomischen Ungleichheit, wenn es auch bedeutsame Unterschiede zwischen den einzelnen Staaten, Regionen und Gruppen gibt (vgl. Salverda 2014). Obwohl die staatlichen Umverteilungseffekte durch die Austeritätspolitik generell abnahmen, sind nach wie vor die institutionellen Leistungen der verschiedenen Systeme der sozialen Sicherung wichtige Bestimmungsfaktoren für Unterschiede in der Entwicklung der Ungleichheit in den einzelnen Ländern (vgl. OECD 2011).

Die Feststellung von Vermögensunterschieden ist auf Grund der Differenzen der Berechnungsweise und der Verfügbarkeit der Daten schwierig, aber alle Untersuchungen von OECD, Oxfam bis Merrill Lynch lassen erkennen, dass der Anstieg der Vermögen noch stärker war als jener der Einkommen. Er war insbesondere in manchen der emerging markets sehr hoch, aber 
Nordamerika verzeichnet noch immer den größten absoluten Vermögenszuwachs und repräsentiert rund ein Drittel des gesamten globalen Vermögens. In den USA, aber auch in anderen Ländern, ist es zudem zu einer Verlagerung von den reichen zu den sehr reichen Haushalten gekommen (vgl. Stiglitz 2013). Diese Zunahme der Vermögenswerte ist vor allem durch Gewinne auf den Finanzmärkten verursacht und führt dazu, dass die Vermögenskonzentration jene der Einkommenskonzentration weit übersteigt, was auch den Bedeutungsverlust der Arbeitseinkommen gegenüber den Kapitaleinkommen für die Vermögensbildung reflektiert.

\section{Wirtschaftssoziologie und ökonomische Ungleichheit}

Vor der neoliberalen Wende hatte nicht nur in Staaten mit starker Wohlfahrtsausrichtung, sondern auch in Marktgesellschaften ein egalitärer Zeitgeist vorgeherrscht. Dies war nicht so sehr ethisch begründet, sondern durch die Annahme, dass die Anhebung und Sicherung der Masseneinkommen durch die dadurch ausgelösten Nachfragewirkungen das wirtschaftliche Wachstum fördern würden. Das Umschwenken auf Neoliberalismus und eine angebotsorientierte Wachstumspolitik haben dann die Politik und die Wertesysteme der Gesellschaften auf die Rechtfertigung von Unterschied und Ungleichheit umgepolt (vgl. Fraser 1997; Gill 2002), bis hin zur expliziten Legitimierung von Reichtum und Bereicherung (vgl. Fligstein 1997).

Der Anstieg der ökonomischen Ungleichheit führt zu einer Polarisierung von Lebenslagen und Lebenschancen. Auf der einen Seite kommt es zu einem neuen Anwachsen von Problemen der Armut, der Deprivation und der Exklusion, mit Folgen für die Gesundheit, die Ausbildungs- und die Berufschancen. Die Probleme betreffen aber in der Gegenwart nicht mehr nur gesellschaftliche Randgruppen, sondern werden verallgemeinert zu individuellen Risiken des Scheiterns (Castel 1995). Aber nicht nur die Lebenslagen und die sozialen Strukturen verändern sich, denn wirtschaftlicher Wandel erfolgt immer auf der Grundlage von Einstellungen und Wertvorstellungen, wird durch diese ermöglicht und führt seinerseits zu Veränderungen von Denkweisen. Steigende Ungleichheit hat auch Auswirkungen auf die Beziehungen zwischen den Menschen, auf ihre Einstellungen, ihr Vertrauen in ihre soziale Umwelt, in die Politik und ihre Zukunft (vgl. Nolan 2007; Nolan and Whelan 2014).

Jede Wirtschaftskonstellation bedarf zu ihrer Rechtfertigung einer Moral, die sie auch für diejenigen akzeptabel macht, die erwartungsgemäß nicht von ihr profitieren werden. Der Neoliberalismus gründet daher nicht nur in einer Ideologie der Elite, sondern stützt sich auch auf allgemeine Wertvorstellungen, auf Werte der individuellen Leistung und auf die Forderungen nach Selbstverwirklichung, wie sie gerade von den Kritikern des bürokratischen Industrialismus der 60er Jahre erhoben worden waren. Deren Kritik wurde, wie Boltanski und Chiapello (1999) meinen, zu Prinzipien des neuen Managements umfunktioniert. Auf der Ebene der Politik wur- 
den Diskurse über Staatsversagen, über negative Folgen von Umverteilung und von sozialpolitischen Maßnahmen verbreitet sowie auch die Rhetorik der Wissensgesellschaft, der ständigen Innovationen und der Wettbewerbsfähigkeit. Amable spricht von der Etablierung einer Ethik der ,competitiveness“ (Amable 2011), die auf die De-Legitimierung kollektiven Handelns, das auf Umverteilung bzw. den Schutz vor Wettbewerb gerichtet ist, abzielte und den Quietismus angesichts wachsender ökonomischer Disparitäten förderte.

Nach 1980 kam es daher zunächst sogar zu einem Anstieg der Akzeptanz von Ungleichheit in den meisten Ländern (Kelley und Evans 1993), wobei insbesondere die positive Sicht der Ungleichheit in Ländern, die eine Transformation vom Sozialismus zu einem Marktsystem erfahren hatten, bemerkenswert war (Gijsberts 2002; Kelley und Zagorski 2004). Unterschiede zeigten sich auch auf Grund von Erfahrungen, Werten und Normen, die sich in der Vergangenheit gebildet haben (vgl. Austen 1999). So wirkte sich eine lange wohlfahrtsstaatliche Tradition sowie die Erfahrungen sozialer Konflikte oder ökonomischer Krisen auf die Wahrnehmung von Ungleichheit als legitim oder illegitim aus (Hadler 2005; Svallfors 2005).

Mit dem Platzen der Finanzblase von 2008 und der daran anschließenden Rezession, die wieder an die Anfälligkeit des Kapitalismus für Krisen und an die Große Depression um 1930 erinnerte, änderte sich die Akzeptanz von Ungleichheit (Kenworthy and McCall 2008). Zugleich stieg die Erkenntnis, dass die wachsende Ungleichheit nicht einfach passierte, sondern durch politische Entscheidungen und materielle Interessen bestimmter Gruppen gefördert wird (vgl. Stiglitz 2013). Das Vertrauen in den „basic bargain“ (Reich 2010, 28 ff.), den Ausgleich der Interessen, schwand und die Wohlstandserwartungen und die Hoffnungen auf Lebenschancen, die durch die Betonung individueller Leistung in den Menschen durch die Ideologie des Neoliberalismus gefördert worden waren, wurden für viele von ihnen enttäuscht. Dies ließ Kritik entstehen und fördert Konflikte sowie auch politischen Radikalismus, was wieder eine wachsende Instabilität des politischen Systems begünstigt und nicht geeignet ist, Vertrauen zu fördern, Konfrontationen zu vermeiden oder vernünftige wirtschaftspolitische Entscheidungen zu treffen.

Kritiker der Globalisierung hoben hervor, dass es neben ökonomischen Verteilungsunterschieden auch zu Verschiebungen der Machtverhältnisse gekommen ist. Sie stellten die Entstehung von globalen Machteliten fest, die den Globalisierungsprozess vorantreiben und von ihm und von den dynamischen Finanzmärkten profitieren (vgl. Sklair 2001). Sie wurden als eine globale ökonomische Klasse erkannt, deren Einkommen und Vermögen nicht mehr an den Kategorien ihres Herkunftslandes gemessen werden kann, sondern sich nur mehr in globalen Bezügen vergleichen lässt (vgl. Skocpol 2004). Durch ihren Einfluss und ihre Macht können sie politische Maßnahmen, die eine Umverteilung oder eine Änderung des Wirtschaftssystems bewirken könnten, verhindern. Die Asymmetrie der Machtverhältnisse zwischen den neuen globalen Eliten und der Masse jener, die sich in lokalen und nationalen Grenzen den wirtschaftlichen 
Fährnissen ausgeliefert fühlen, verstärkt die Polarisierung durch die ökonomische Ungleichheit. Selbst Angehörige der Mittelschichten entwickeln auf Grund der Ohnmacht, die sie empfinden, ein Unterschichtgefühl (vgl. Neckel 2008).

In globaler Perspektive zeigen sich Auswirkungen auf die nationalen, regionalen und lokalen Strukturen und Lebensbedingungen der Menschen mit erschreckenden Folgen (vgl. Therborn 2013). Das eklatante Wohlstandsgefälle und der Anstieg der Unsicherheit des Lebens in vielen Teilen der Welt lösen auch Migrationsbewegungen aus, wodurch es zu Verschiebungen kommt, die Einfluss auf die weitere Verteilung der Einkommen, Vermögen, Zugangsmöglichkeiten, Lebensbedingungen etc. haben (Sassen 2005). Das birgt oder verstärkt auch das Potential für neue gesellschaftliche Konflikte mit einer globalen räumlichen Dimension und großer politisch-kultureller Brisanz. Wirtschaftliche Verhältnisse, soziale und ökonomische Probleme verknüpfen sich mit ethnischen, religiösen und ideologischen Differenzen, weltpolitischen Machtkonflikten und sozialstrukturellen Disparitäten.

In der Gegenwart kann sich die Wirtschaftssoziologie angesichts dieser Entwicklungen weder nur auf die Erhebung von Denk- und Verhaltensmustern von Arbeitenden oder Konsumenten noch auf die Einbettung der Wirtschaft in soziale Beziehungen und Institutionen beschränken. Neben den Fragen der Koordination wirtschaftlicher Aktivitäten, muss auch den Problemen der Verteilung vermehrt Beachtung geschenkt werden, sind diese doch, wie Beckert erkennt, ebenso „[...] eminent soziologische Fragen [...]“ (Beckert 2009b, S. 18). Wie schon in der Zeit der Herausbildung des industriellen Kapitalismus und der Klassiker der Wirtschaftssoziologie muss sie sich mit den gesellschaftlichen und kulturellen Folgen der ökonomischen Veränderungen auseinandersetzen. Dabei geht es aber nicht mehr um deren Deutung als Rationalisierungs-, Individualisierungs- oder Modernisierungsprozess, sondern darum, Wirtschaft als politisch-kulturellen Tatort zu begreifen und aus soziologischer Problemsicht neu zu deuten. Inmitten von andauernden Krisen muss es wieder zur Einsicht kommen, dass Wirtschaft von allen und für alle gemacht wird.

\section{Literatur}

Albert, Hans. 2014. Nationalökonomie als Soziologie der kommerziellen Beziehungen. Ursprl. 1956. Tübingen: Mohr (Paul Siebeck).

Amable, Bruno. 2011. Morals and Politics in the Ideology of Neo-Liberalism. Socio-Economic Review 9: 3-130.

Aspers, Patrick. 2011. Markets. Cambridge, UK-Malden, MA: Polity Press.

Arrighi, Giovanni. 2002. The Long Twentieth Century. Money, Power, and the Origins of Our Times. London-New York: Verso. 
Atkinson, Anthony B. 2007. The distribution of earnings in OECD countries. International Labour Review 146 (1-2): 41-60.

Atkinson, Anthony B., Thomas Piketty and Emmanuel Saez. 2011. Top Incomes in the Long Run of History. Journal of Economic Literature 49 (1): 3-71.

Austen, Siobhan. 1999. Norms of Inequality. Journal of Economic Issues XXXIII (2): 435-442.

Beckert, Jens. 1996. Was ist soziologisch an der Wirtschaftssoziologie? Ungewissheit und die Einbettung wirtschaftlichen Handelns. Zeitschrift für Soziologie 25(2): 125-146.

Beckert, Jens. 2009a. Wirtschaftssoziologie als Gesellschaftstheorie. Zeitschrift für Soziologie 38(3): 182197.

Beckert, Jens. 2009b. Koordination und Verteilung. Zwei Ansätze der Wirtschaftssoziologie. In: Die Ökonomie der Gesellschaft, hrsg. Sylke Nissen und Georg Vobruba. 17-34. Wiesbaden: VS Verlag.

Beckert, Jens, Rainer Diaz-Bone und Heiner Ganßmann. 2007. Märkte als soziale Strukturen. Frankfurt a.M.: Campus Verlag.

Bögenhold, Dieter. 2011. Sozioökonomik: Das Verhältnis von Wirtschaft und Gesellschaft akademisch betrachtet. In: Sozioökonomie: Die Rückkehr der Wirtschaft in die Gesellschaft, hrsg. Gertraude MiklHorke, 59-94. Marburg: Metropolis.

Bologna, Sergio. 2006. Die Zerstörung der Mittelschichten. Thesen zur neuen Selbständigkeit. Graz: Nausner \& Nausner.

Boltanski, Luc et Eve Chiapello. 1999. Le nouvel ésprit du capitalisme. Paris: Gallimard.

Bourdieu, Pierre. 1992. Die feinen Unterschiede. Frz.Orig.v. 1979. Frankfurt/Main: Suhrkamp.

Burt, Ronald S. 1992. Structural Holes: the Social Structure of Competition. Cambridge, MA: Harvard Univ. Press.

Castel, Robert. 1995. Les métamorphoses de la question sociale. Paris: Fayard.

Coleman, James S. 1993. The Rational Reconstruction of Society. American Sociological Review 58: 1-15. Deutschmann, Christoph, 2008. Kapitalistische Dynamik. Eine gesellschaftstheoretische Perspektive. Wiesbaden: VS Verlag für Sozialwissenschaften.

Fligstein, Neil. 1997. Markets, Politics, and Globalization. Uppsala: Uppsala University Press.

Fraser, Nancy. 1997. Justice Interruptus: Critical Reflections on the "Postsocialist" Condition. New YorkLondon: Routledge.

Fürstenberg, Friedrich. 1966. „Sozialstruktur“ als Schlüsselbegriff der Gesellschaftsanalyse. Kölner Zeitschrift für Soziologie und Sozialpsychologie 18: 439-453.

Ganßmann, Heiner, 1996. Geld und Arbeit: Wirtschaftssoziologische Grundlagen einer Theorie der modernen Gesellschaft. Frankfurt: Campus.

Gijsberts, Mérove. 2002. The Legitimation of Income Inequality in State-socialist and Market Societies. Acta Sociologica 45(4): 269-285.

Gill, Stephen. 2002. Constitutionalizing inequality and the clash of globalizations. International Relations Review 4(2): 47-65. 
Goesling, Brian. 2001. Changing Income Inequalities Within and Between Nations: New Evidence. American Sociological Review 66(5): 745-761.

Granovetter, Mark. 1985. Economic Action and Social Structure: The Problem of Embeddedness. American Journal of Sociology 91: 481-510.

Granovetter, Mark. 2005. The Impact of Social Structure on Economic Outcomes. Journal of Economic Perspectives 19: 33-50.

Hadler, Markus. 2005. Why Do People Accept Different Income Ratios? A Multi-level Comparison of Thirty Countries. Acta Sociologica 48(2): 131-154.

Kelley, Jonathan and M. D. R. Evans. 1993. The Legitimation of Inequality: Occupational Earnings in Nine Nations. American Journal of Sociology 99(1): 75-126.

Kelley, Jonathan and Krzysztof Zagorski. 2004. Economic Change and the Legitimation of Inequality: The Transition from Socialism to the Free Market in Central-East Europe. Research in Social Stratification and Mobility 22: 319-364.

Kenworthy, Lane and Leslie McCall. 2008. Inequality, Public Opinion and Redistribution. Socio-Economic Review 6: 35-68.

Kreckel, Reinhard. 1998. Klassentheorie am Ende der Klassengesellschaft. In: Alte Ungleichheiten - neue Spaltungen, hrsg. Peter A. Berger und Michael Vester. 31-47. Opladen: Leske \& Budrich.

Maurer, Andrea und Gertraude Mikl-Horke. 2015. Wirtschaftssoziologie. Baden-Baden: UTB Nomos.

Mikl-Horke, Gertraude. 2008. Sozialwissenschaftliche Perspektiven der Wirtschaft. München-Wien: O1denbourg.

Mikl-Horke, Gertraude. 2011. Sozioökonomie: Die Rückkehr der Wirtschaft in die Gesellschaft. Marburg: Metropolis.

Mikl-Horke, Gertraude. 2014. Geld oder Leben. Reflexionen über Wirtschaft und Gesellschaft in Zeiten der Finanzmarktdominanz. In: Sozioökonomische Perspektiven. Texte zum Verhältnis von Gesellschaft und Ökonomie, hrsg. Gerda Bohmann, Johanna Hofbauer und Johann August Schülein, 17-58. Wien: facultas-wuv.

Myles, John. 2003. Where Have All the Sociologists Gone? Explaining Economic Inequality. Canadian Journal of Sociology 28(4): 551-559.

Neckel, Sighart. 2008. Die gefühlte Unterschicht. Vom Wandel der sozialen Selbsteinschätzung. In: Unterschicht, hrsg. Rolf Lindner und Lutz Musner, 19-40. Freiburg i.Br.: Raubach.

Nolan, Brian. 2007. A Comparative Perspective on the Development of Poverty and Exclusion in European Societies. International Policy Analysis. Berlin: Friedrich Ebert-Stiftung. http://library.fes.de/ pdf-files/id/05016.pdf

Nolan, Brian and Christopher T. Whelan. 2014. The Social Impact of Income Inequality: Poverty, Deprivation, and Social Cohesion. In: Changing Inequalities in Rich Countries. Analytical and Comparative Perspectives, hrsg. Salverda, Wiemer et al., 146-168. Oxford: Oxford University Press.

Nollmann, Gerd. 2007. Sozialstruktur und Gesellschaftsanalyse. Wiesbaden: Springer VS.

OECD. 2011. Divided We Stand: Why Inequality Keeps Rising. Paris: OECD. 
Ötsch, Walter Otto. 2009. Mythos Markt. Marktradikale Propaganda und ökonomische Theorie. Marburg: Metropolis.

Parsons, Talcott and Neil J. Smelser. 1956. Economy and Society. London: Routledge \& Kegan.

Preglau, Max. 2007. Soziologische Gesellschaftsanalyse und Politikwissenschaft. In: Polarisierte Gesellschaft? hrsg. Thomas Geiger, Martin Hartlieb and Birgit Winkel, 260-270. Innsbruck: Studienverlag.

Reich, Robert B. 2010. Aftershock. The Next Economy and America's Future. New York: Alfred A. Knopf.

Salverda, Wiemer et al. 2014. Changing Inequalities in Rich Countries. Analytical and Comparative Perspectives. Oxford: Oxford University Press.

Sassen, Saskia. 2005. New Global Classes: Implications for Politics. In: The New Egalitarianism. hrsg. Anthony Giddens and Patrick Diamond, 143-153 Cambridge. UK-Malden, MA: Polity Press.

Schmid, Michael und Andrea Maurer. 2003. Ökonomischer und soziologischer Institutionalismus. Marburg: Metropolis.

Sklair, Leslie. 2001. The Transnational Capitalist Class. Malden, MA-Oxford, UK: Blackwell.

Skocpol, Theda. 2004. Civic Transformation and Inequality in the Contemporary United States. In: Social Inequality, hrsg. Kathyrn M. Neckerman, 729-767. New York: Russell Sage Foundation.

Stiglitz, Joseph E. 2013. The Price of Inequality. London et al.: Penguin.

Svallfors, Stefan. 2005. Analyzing Inequality: Life Chances and Social Mobility in Comparative Perspective. Stanford, CA: Stanford University Press.

Svallfors, Stefan. 2006. The Moral Economy of Class: Class and Attitudes in Comparative Perspective. Stanford, CA: Stanford University Press.

Therborn, Göran. 2013. The Killing Fields of Inequality. Cambridge, MA: Polity Press.

Weber, Max. 2000. Die Börse. Zweck und äußere Organisation der Börsen (1894). In: Max Weber-Gesamtausgabe. Abt. I, Bd. 5, hrsg. Knut Borchardt und C. Mayer-Stoll, 135-174. Tübingen: Mohr (Siebeck). White, Harrison C. 1981. Where Do Markets Come from? American Journal of Sociology 87: 517-547.

Windolf, Paul. 2005. Finanzmarkt-Kapitalismus. Analysen zum Wandel von Produktionsregimen. Wiesbaden: VS-Verlag für Sozialwissenschaften.

Gertraude Mikl-Horke, geb. 1944 in Wien, studierte Handelswissenschaften und war nach einem mehrjährigen Arbeitsaufenthalt in Japan und der Habilitation für Allgemeine Soziologie und Wirtschaftssoziologie von 1981 bis 2009 Professorin an der Wirtschaftsuniversität Wien. Forschungsbereiche: Arbeitsund Industriesoziologie, Theorien der Wirtschaftssoziologie, Wissenschaftsgeschichte der Soziologie und Ökonomie, japanische Wirtschaftsgesellschaft. Zahlreiche Publikationen und Beiträge in internationalen Zeitschriften, zuletzt: zus. mit Andrea Maurer, Wirtschaftssoziologie, UTB-Nomos: Baden-Baden 2015. 
